(12/47), 20\% of torcular (3/15), and $8 \%$ of cavernous sinus (5/60) dAVFs. Of 68 dAVFs with APA supply, embolization was carried out via this pedicle in eight (12\%), and seven were ultimately occluded.There were no complications, including no post-treatment cranial neuropathies or radiographic evidence of nontarget embolization. For five dAVFs, the APA was selected as the initial pedicle for embolization (marginal sinus, $\mathrm{n}=2$; distal sigmoid, $\mathrm{n}=1$; cavernous, $\mathrm{n}=1$, tentorial, $\mathrm{n}=1)$. In $4 / 5$ cases, dAVF occlusion was achieved via the initial APA feeding artery pedicle. In one case, near complete, stagnant occlusion was achieved; adjunctive embolization of a single additional MMA pedicle was performed. In three other cases of complex transverse/sigmoid dAVF, the APA was utilized after multiple attempts via middle meningeal and occipital artery pedicles. Occlusion was not achieved transarterially; two of the three dAVFs were ultimately occluded transvenously.

Conclusion In rare, select cases, the APA is an excellent route for transarterial embolization of cranial dAVFs.

Disclosures B. Gross: None. F. Albuquerque: None. K. Moon: None. C. McDougall: None.

\section{P-023 CEREBRAL ARTERIOVENOUS MALFORMATION FLOW IS ASSOCIATED WITH VENOUS INTIMAL HYPERPLASIA}

${ }^{1} \mathrm{~S}$ Shakur, ${ }^{1} \mathrm{~A}$ Hussein, ${ }^{1} \mathrm{~S}$ Amin-Hanjani, ${ }^{2} \mathrm{~T}$ Valyi-Nagy, ${ }^{1} \mathrm{~V}$ Aletich, ${ }^{1} \mathrm{~F}$ Charbel, ${ }^{1} \mathrm{~A}$ Alaraj. ${ }^{1}$ Neurosurgery, University of Illinois at Chicago, Chicago, IL; ${ }^{2}$ Pathology, University of Illinois at Chicago, Chicago, IL

\subsection{6/neurintsurg-2016-012589.65}

Introduction/purpose Histopathological changes in cerebral arteriovenous malformation (AVM) draining veins secondary to chronically high AVM inflow have not been clearly elucidated. Here, we examine the relationship between draining vein wall thickness and AVM flow rate.

Materials and methods Records of patients with cerebral AVMs evaluated at our institution between 2007-2013 were retrospectively reviewed. Patients were included if a surgical specimen of the nidus was available and if flows were obtained before treatment using quantitative magnetic resonance angiography. Specimens were mounted on slides and stained with hematoxylin and eosin as well as elastin special stain. Perinidal veins were identified and the wall thickness of each vein was measured from digitized images of the slides (Figure 1). Maximum vein wall thickness was recorded for each specimen.

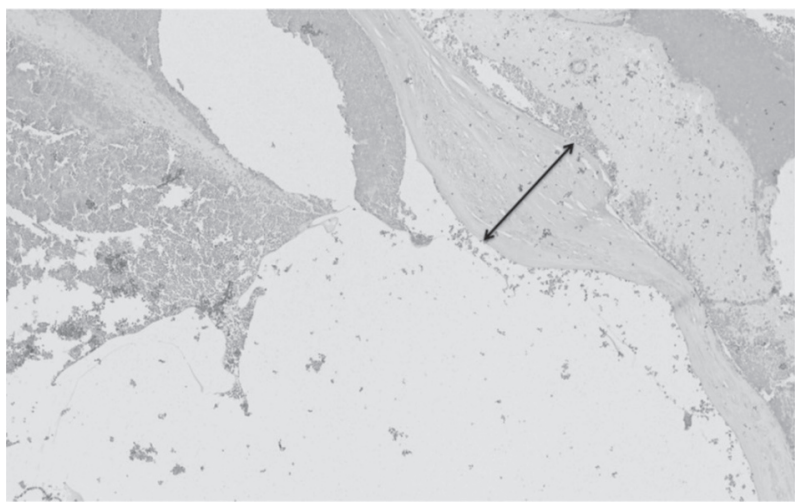

Abstract P-023 Figure 1 Example of a perinidal vein with thickened wall. Elastin special stain; original magnification $\times 10$

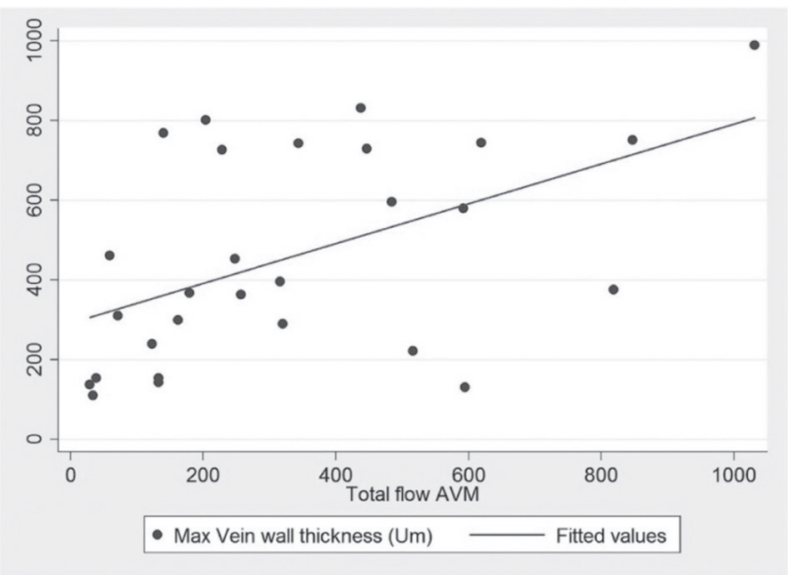

Abstract P-023 Figure 2 Maximum vein wall thickness $(\mu \mathrm{m})$ versus total AVM flow (mL/minute) (rho $=+0.51, P=0.006$ )

Intranidal arteries were also identified and the diameter of each artery was measured. Total AVM flow was estimated as aggregate flow within primary arterial feeders or flow in single draining veins. The relationship between maximum vein wall thickness, total AVM flow, flow per draining vein, flow per unit volume of AVM, and mean intranidal artery diameter was assessed.

Results 28 patients (20 male, 8 female) with mean age of 37 years (range 16-68 years) were included. Spearman's correlation revealed a statistically significant relationship between maximum vein wall thickness and total AVM flow (rho $=+0.51, P=0.006$ ) (Figure 2) as well as AVM flow per draining vein (rho $=+0.41, P=0.03$ ). However, there was no statistically significant correlation between maximum vein wall thickness and flow per unit volume of AVM (rho $=+0.27, P=0.17$ ) or mean intranidal artery diameter (rho $=+0.42, P=0.24$ ). Mean vein wall thickness was significantly higher in the presence of venous ectasia $(562 \mu \mathrm{m}$ vs. $300 \mu \mathrm{m}, P=0.007)$. Presence of venous stenosis was not significantly associated with age, Spetzler-Martin grade, volume, number of draining veins, deep venous drainage, intranidal fistula, or maximum vein wall thickness.

Conclusion Maximum vein wall thickness is significantly related to total AVM flow and AVM flow per draining vein. This finding implicates chronically high AVM inflow in venous intimal hyperplasia and possible subsequent development of venous outflow stenosis.

Disclosures S. Shakur: None. A. Hussein: None. S. Amin-Hanjani: None. T. Valyi-Nagy: None. V. Aletich: None. F. Charbel: None. A. Alaraj: None.

\section{P-024 PROMINENT CONDYLAR VEINS CAUSING PULSATILE TINNITUS: DYNAMIC ANGIOGRAPHIC CONFIRMATION}

${ }^{1} \mathrm{M}$ Alexander, ${ }^{2} \mathrm{~K}$ Meisel, ${ }^{1} \mathrm{~V}$ Halbach, ${ }^{1} \mathrm{R}$ Darflinger, ${ }^{1} \mathrm{~A}$ Nicholson, ${ }^{1} \mathrm{~F}$ Settecase, ${ }^{1} \mathrm{D}$ Cooke, ${ }^{1} \mathrm{R}$ Higashida, ${ }^{1} \mathrm{C}$ Dowd, ${ }^{1} \mathrm{~S}$ Hetts, ${ }^{1} \mathrm{M}$ Amans. ${ }^{1}$ Radiology and Biomedical Imaging, UCSF, San Francisco, CA; ${ }^{2}$ Neurology, UCSF, San Francisco, CA

\subsection{6/neurintsurg-2016-012589.66}

Introduction/purpose Numerous processes can cause pulsatile tinnitus (PT), some of which are potentially life threatening. This case series describes a cause of PT - prominent condylar veins - that has undergone little investigation to date. This report characterizes angiographic findings in patients with 